\title{
Successful Treatment of Corticosteroid with Antiviral Therapy for a Neonatal Liver Failure with Disseminated Herpes Simplex Virus Infection
}

\author{
Shinji Maeba, MD, $\mathrm{PhD}^{1, *}$ Shunji Hasegawa, MD, $\mathrm{PhD}^{1, *}$ Maiko Shimomura, MD, $\mathrm{PhD}^{1}$ \\ Takuya Ichimura, $\mathrm{MD}^{1}$ Kazumasa Takahashi, MD, $\mathrm{PhD}^{1}$ Masashi Motoyama, MD, $\mathrm{PhD}^{1}$ \\ Shinnosuke Fukunaga, MD, $\mathrm{PhD}^{1}$ Yoshinori Ito, MD, $\mathrm{PhD}^{2}$ Takashi Ichiyama, MD, $\mathrm{PhD}^{1,3}$ \\ Shouichi Ohga, MD, $\mathrm{PhD}^{1}$
}

\footnotetext{
${ }^{1}$ Department of Pediatrics, Yamaguchi University Graduate School of Medicine, Yamaguchi, Japan

2 Department of Pediatrics, Nagoya University Graduate School of Medicine, Aichi, Japan

${ }^{3}$ Division of Pediatrics, Tsudumigaura Medical Center for Children With Disabilities, Yamaguchi, Japan
}

Address for correspondence Shunji Hasegawa, MD, PhD, Department of Pediatrics, Yamaguchi University Graduate School of Medicine, 1-1-1 Minamikogushi, Ube, Yamaguchi 755-8505, Japan (e-mail: shunji@yamaguchi-u.ac.jp).

\section{Abstract \\ Keywords \\ - fulminant liver failure \\ - newborn \\ - herpes simplex virus \\ - acyclovir \\ - methylprednisolone pulse therapy}

Background Herpes simplex virus (HSV) infection carries one of the poorest outcomes of neonatal liver failure (NLF). Neonates with disseminated HSV infection can develop hemophagocytic lymphohistiocytosis $(\mathrm{HLH})$, and occasionally need orthotopic liver transplantation. Early interventions may be critical for the cure of NLF.

Case Report We describe herewith a 6-day-old neonate with fulminant hepatic failure due to disseminated HSV-1 infection, who successfully responded to high-dose corticosteroid therapy 72 hours after the onset of disease. Preceding acyclovir, gamma globulin, and exchange blood transfusion therapies failed to control the disease. Methylprednisolone pulse therapy led to a drastic improvement of liver function and cytokine storms, and prevented the disease progression to $\mathrm{HLH}$. Sustained levels of plasma and cerebrospinal fluid HSV DNA declined after prolonged acyclovir therapy. Bilateral lesions of the periventricular white matter areas, assessed by magnetic resonance imaging, disappeared at 3 months of age. The infant showed normal growth and development at 4 years of age.

Conclusion Early anti-hypercytokinemia therapy using corticosteroid, and prolonged antiviral therapy might only provide the transplantation-free cure of NLF with HSV dissemination.

\section{Clinical Perspective}

Early anti-hypercytokinemia therapy using corticosteroid and prolonged antiviral therapy might only provide the

S. Maeba and S. Hasegawa contributed equally to this article. transplantation-free cure of neonatal liver failure with HSV dissemination.

Neonatal liver failure (NLF), occurring as fulminant hepatic failure within the first 28 days of birth, is rare, but has a high mortality. ${ }^{1}$ It arises from various etiologies including received

June 6,2014

accepted after revision

August 12, 2014

published online

April 6, 2015
DOI http://dx.doi.org/

10.1055/s-0034-1398392. ISSN $2157-7005$.
Copyright $\odot 2015$ by Thieme Medical Publishers, Inc., 333 Seventh Avenue, New York, NY 10001, USA. Tel: +1(212) 584-4662.
License terms

(요 (1) $\Theta \circledast$ 
neonatal hemochromatosis, inborn errors of metabolism, infections, and hemophagocytic lymphohistiocytosis (HLH). Herpes simplex virus (HSV) infection is one of the major causes of acute liver failure $e^{2,3}$ and acquired HLH in early infancy. ${ }^{4}$ HSV-1 accounts for more than $60 \%$ of herpes meningoencephalitis cases in the newborn, which is mostly associated with virus dissemination. The pathophysiology of neonatal HSV infection is explained by systemic inflammatory response syndrome (SIRS), ${ }^{5}$ often progress to liver and respiratory failures, ${ }^{6}$ and the HLH. The survival rate of NLF patients was reportedly lower than that of the other types of NLF patients even after liver transplantation. ${ }^{1}$ Poor neurodevelopmental outcomes occur in approximately 20\% of survivors of disseminated HSV disease, and $70 \%$ of those with central nervous system (CNS) disease. Prolonged acyclovir therapy is the standard for neonatal HSV disease. ${ }^{7}$ However, the effective measures to control HSV-driven NLF and SIRS remains elusive. Corticosteroid therapy is not the rule of NLF management, ${ }^{1}$ particularly in cases with the virus dissemination.

We herein present the case of a 6-day-old neonate with NLF and hypercytokinemia because of disseminated HSV infection. This condition was successfully controlled after methylprednisolone pulse therapy started within 72 hours of the disease onset. Early corticosteroid therapy and prolonged antiviral therapy resulted in a significant reduction of serum cytokine levels and the quantity of viral DNA, respectively. Anti-hypercytokinemia therapy in NLF was then discussed.

\section{Case Report}

A 2,746-g male neonate was born to a healthy mother by vaginal delivery at 39 weeks of gestation, without asphyxia. The infant was transferred to the neonatal intensive care unit 6 days after birth, for fever $\left(38.2^{\circ} \mathrm{C}\right)$, poor feeding, apnea, and lethargy without vesicular skin lesions. On admission, mild dyspnea needed respiratory support by nasal continuous positive airway pressure. Computed tomography indicated brain edema. Results of hematological examinations were as follows: leukocytes, $5.15 \times 10^{9} / \mathrm{L}$; hemoglobin, 14.3 $\mathrm{g} / \mathrm{dL}$; platelets, $148 \times 10^{9} / \mathrm{L}$; C-reactive protein, $2.99 \mathrm{mg} / \mathrm{dL}$ (reference range $[\mathrm{rr}] ;<0.3 \mathrm{mg} / \mathrm{dL}$ ); alanine aminotransferase (ALT), $455 \mathrm{IU} / \mathrm{L}$ (rr, 5-43 IU/L); aspartate aminotransferase (AST), 2,220 IU/L (rr, 12-34 IU/L); lactic dehydrogenase (LDH), 7,510 IU/dL (rr, 115-217 IU/dL); direct bilirubin, 1.3 $\mathrm{mg} / \mathrm{dL}$ (rr, 0.1-0.3 mg/dL), cholesterol, 78mg/dL (rr, 130-220 $\mathrm{mL} / \mathrm{dL}$ ); triglyceride, $33 \mathrm{mg} / \mathrm{dL}$ ( $\mathrm{rr}, 46-130 \mathrm{mg} / \mathrm{dL}$ ); ferritin, $141,066 \mathrm{ng} / \mathrm{mL}$ (rr, 25.0-280.0 ng/mL); prothrombin time (PT), 15.8 seconds; PT-international normalized ratio (PT-INR), 1.79; fibrinogen, $209 \mathrm{mg} / \mathrm{dL}$ ( $\mathrm{rr}, 200-310 \mathrm{mg} /$ $\mathrm{dL}$ ); and D-dimer, $33.6 \mathrm{mg} / \mathrm{L}$ ( $\mathrm{rr}, 0-1 \mathrm{mg} / \mathrm{L}$ ). Cerebrospinal fluid (CSF) examinations revealed leukocytes, 2 cells $/ \mu \mathrm{L}$ ( $\mathrm{rr},<5$ cells/ $\mu \mathrm{L})$; glucose, $52 \mathrm{mg} / \mathrm{dL}(\mathrm{rr}, 60-80 \mathrm{mg} / \mathrm{dL})$; and protein, $64 \mathrm{mg} / \mathrm{dL}$ ( $\mathrm{rr}, 15-45 \mathrm{mg} / \mathrm{dL}$ ). Under the tentative diagnosis of sepsis and hepatitis, antibiotics and acyclovir were started $(60 \mathrm{mg} / \mathrm{kg} / \mathrm{d})$. Laboratory examinations performed 8 hours later indicated progressive liver damage with disseminated intravascular coagulation; ALT, $884 \mathrm{IU} / \mathrm{L}$; AST, 4,459 IU/L; LDH, 13,746 IU/dL; platelets, $104 \times 10^{9} / \mathrm{L}$, PT, 22.4 seconds; $\quad$ PT-INR, 3.11; fibrinogen, $114 \mathrm{mg} / \mathrm{dL}$; and D-dimer, $37.4 \mathrm{mg} / \mathrm{L}$. Infusions of fresh frozen plasma, and whole exchange blood transfusions were instituted. High copy numbers of HSV-1 DNA, but not, the other types of herpes viruses DNA on admission (serum, $1.8 \times 10^{7}$ copies $/ \mathrm{mL}$; CSF, $5.7 \times 10^{5}$ copies $/ \mathrm{mL}$, assessed by realtime polymerase chain reaction ${ }^{8}$ ) confirmed the diagnosis of NLF because of disseminated HSV-1 infection. Repeated exchange blood transfusions failed to control liver function and coagulopathy (-Fig. 1A). Additional high-dose immunoglobulin therapy $(2 \mathrm{~g} / \mathrm{kg} / \mathrm{d} \times 2 \mathrm{~d})$ on 8 and 9 days after birth was ineffective. Then, methylprednisolone therapy (30 $\mathrm{mg} / \mathrm{kg} / \mathrm{d} \times 3 \mathrm{~d}$ ) led to drastic improvement of coagulopathy (PT-INR), declining transaminase levels, and defervescence. Transaminase levels peaked on the 2nd day of treatment (AST: 5,394 IU/L; ALT: $980 \mathrm{IU} / \mathrm{L}$ ), and no any organ failure developed. The fluid-attenuated inversion recovery (FLAIR) magnetic resonance imaging (MRI) 15 days after birth showed multiple high-signal lesions in the white matter (-Fig. 2A). Acyclovir therapy was continued until 43 days after birth. The copy number of CSF HSV DNA decreased to $2.9 \times 10^{2} \mathrm{copy} / \mathrm{mL} 30$ days after birth. The serum copy numbers steadily decreased and became undetectable 49 days after birth (-Fig. 1B). No HSV infection recurred after the hospital discharge. Funduscopy, automated auditory brain stem response, and electroencephalogram findings were all normal. FLAIR MRI at 3 months (-Fig. 2B) and 2 years and 9 months of age revealed no abnormalities. At the age of 4 years, he is alive and well having 90 score of

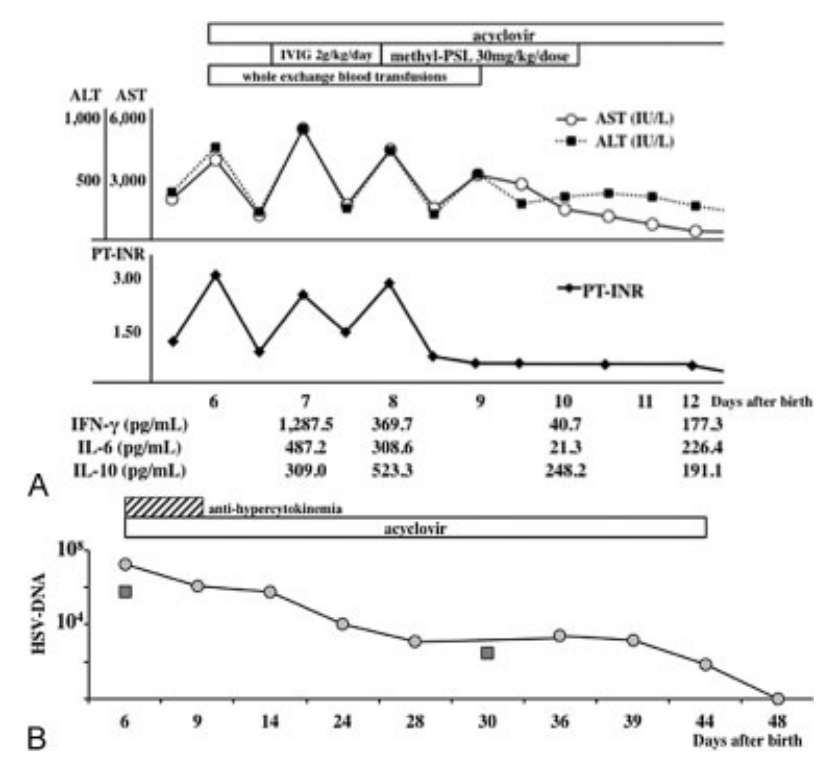

Fig. 1 Sequential changes of the liver function and serum cytokine levels during the initial treatment course $(A)$, and those of HSV DNA copy number in serum $(\bullet)$ and cerebrospinal fluid $(\boldsymbol{\square})$ during the overall treatment course (B). ALT, alanine aminotransferase; AST, aspartate aminotransferase; IVIG, intravenous high-dose immunoglobulin therapy; HSV: herpes simplex virus; IFN, interferon; IL, interleukin; PT-INR, prothrombin time-international normalized ratio. 

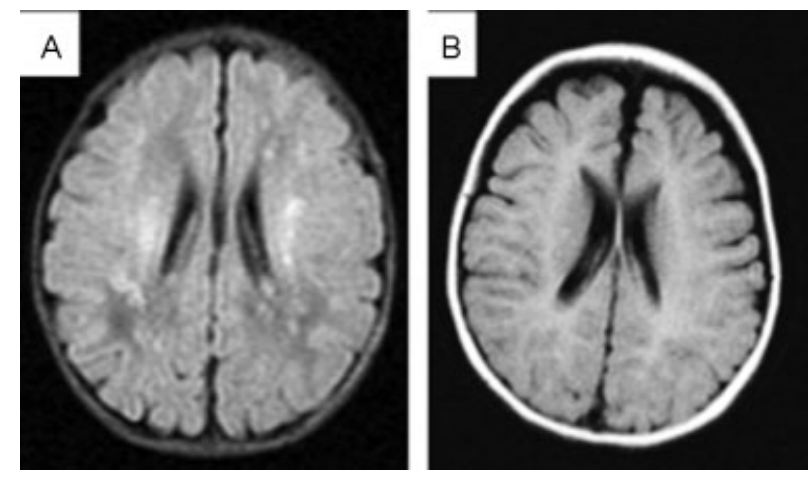

Fig. 2 Head magnetic resonance imaging (fluid-attenuated inversion recovery) scan shows multiple high-signal lesions in the white matter at 15 days of age (A), and no abnormalities at 3 months of age (B).

intelligence quotient assessed by the Tanaka-Binet intelligence test.

Serum cytokine levels during the acute phase of HSV-NLF (6-12 days after birth) were retrospectively measured by flow cytometry using a cytometric bead array kit (BD Pharmingen, San Diego, CA) according to the manufacturer's instructions. ${ }^{9}$ Seven days after birth, serum levels of interferon (IFN)- $\gamma$, interleukin (IL)-6, and IL-10 were remarkably elevated (- Fig. 1B). On the contrary, CSF cytokine levels on admission were all undetectable. Serum levels of IFN- $\gamma$ and IL-6, but not, IL-10 showed the nadir (10 days after birth) after methylprednisolone therapy, in concert with the full recovery of PT-INR.

\section{Discussion}

Early determination of the causes of NLF is challenging. The survival of affected infants depends on the management according to the progression of metabolic diseases, neonatal hemochromatosis, and primary/secondary HLH. Liver transplantation and hematopoietic stem cell transplantation are the curative treatment at present for neonatal hemochromatosis and familial HLH, respectively. On the contrary, the treatment strategy of HSV-NLF and its-associated HLH has not been established. In this patient, liver dysfunction and CNS disease prompted us to start the acyclovir. Higher copy number of HSV-1 DNA in the serum than seen in CSF indicated disseminated, rather than isolated CNS, infection of the virus. At that time point, the patient fulfilled the diagnostic criteria of $\mathrm{NLF}^{2}$ but not HLH in the newborn. ${ }^{4}$ Corticosteroid therapy is not recommended for the treatment of fulminant hepatic failure. Etoposide therapy does not raise the survival rate of neonatal HSV-associated HLH. Then, methylprednisolone pulse therapy was introduced to the infant for the control of a cytokine storm. Neonatal disseminated infections with HSV-1 or HSV-2 led to SIRS represented by high IL-6 and TNF- $\alpha$ levels, and occasionally to the rapid demise of multiple organ dysfunction syndrome or $\mathrm{HLH}^{9-12}$ Methylprednisolone therapy might be effective for the control of neonatal disseminated HSV infection with HLH. ${ }^{10,11}$ In this reported case, ${ }^{10}$ sequential changes in the declining pattern of IL-6,
IL-10, and IFN- $\gamma$ levels were consistent with the results of our observation, although HSV DNA copy number was not monitored. On the contrary, appreciable levels of IL-10 and IFN- $\gamma$ in the neonates with virus-associated HLH might be associated with a good prognosis. ${ }^{9,10}$ Nagamori et al $^{11}$ have recently reported a successfully treated case of neonatal disseminate HSV infection with early anti-inflammatory therapy using prednisolone. On the basis of the kinetics of high-mobility group box protein1 (HMGB-1) and cytochrome $c$, they concluded that anti-inflammatory intervention may only be effective if it is undertaken during the early phase of disseminated neonatal HSV infections. Uncontrolled coagulopathy portends an ominous outcome of liver failure. In this line, sequential changes in the PT-INR and cytokine levels after early corticosteroid therapy might represent the improvement of liver damages through the controlled hypercytokinemia.

The neonates with HSV-associated HLH (HSV-HLH) sometimes show a lack of several parameters of diagnostic criteria of HLH. ${ }^{4}$ Serum levels of IL-10 and IFN- $\gamma$ were elevated in the present patient, differently from nonHSV-HLH in the newborns. ${ }^{9,10}$ The distinct cytokine profiles may explain the clinical expressions and severity of neonatal HLH, according to the causative agents and genetic predispositions of HLH.

The present patient showed the high quantity of HSV DNA but not cytokines in CSF at the diagnosis. Multiple high-signal lesions in the brain MRI images disappeared with the declining viral loads in the CSF and systemic circulation. The declining pattern of the viral loads was much slower than that of circulating cytokines. After the prolonged acyclovir therapy until the attainment of undetectable HSV DNA, the patient showed neither recurrence of disease nor neurological sequelae. Recently, Scoble and Underwood ${ }^{12}$ reported a 9-day-old neonate with HSV-2 driven NLF and shock, who presented bilateral periventricular lesions on MRI. The white matter lesions disappeared during the convalescent phase of the disease, similarly in our patient. In this context, the brain lesions during the acute phase of HSV-NLE might represent the focal edema and/or hemorrhage through hypercytokinemia. This observation may emphasize the practical need of early cytokine control under acyclovir therapy, for the complete cure of neonatal disseminated HSV infection, to prevent not only the development of $\mathrm{HLH} /$ multiple organ dysfunction syndrome but also the impending CNS involvement. Further study should be focused on the targeted therapy of anti-hypercytokinemia in the newborn neonates with HSV dissemination.

Conflicts of Interest

The authors have no conflicts of interest to disclose.

\section{Acknowledgments}

We thank M. T. Midori Wakabayashi (Department of Pediatrics, Yamaguchi University Graduate School of Medicine, Japan) for the technical support. This work was partly supported by a Grant-in-Aid for Scientific Research from the Ministry of Education, Culture, Sports, Science 
and Technology of Japan, and from the Ministry of Health, Labor and Welfare of Japan.

\section{References}

1 Shanmugam NP, Bansal S, Greenough A, Verma A, Dhawan A. Neonatal liver failure: aetiologies and management-state of the art. Eur J Pediatr 2011;170(5):573-581

2 Verma A, Dhawan A, Zuckerman M, Hadzic N, Baker AJ, MieliVergani G. Neonatal herpes simplex virus infection presenting as acute liver failure: prevalent role of herpes simplex virus type I. J Pediatr Gastroenterol Nutr 2006;42(3):282-286

3 McGoogan KE, Haafiz AB, González Peralta RP. Herpes simplex virus hepatitis in infants: clinical outcomes and correlates of disease severity. J Pediatr 2011;159(4):608-611

4 Suzuki N, Morimoto A, Ohga S, Kudo K, Ishida Y, Ishii E. HLH/LCH Committee of the Japanese Society of Pediatric Hematology. Characteristics of hemophagocytic lymphohistiocytosis in neonates: a nationwide survey in Japan. J Pediatr 2009;155(2): 235-238, e1

5 Kawada J, Kimura H, Ito Y, et al. Evaluation of systemic inflammatory responses in neonates with herpes simplex virus infection. J Infect Dis 2004;190(3):494-498
6 Capretti MG, Marsico C, Lazzarotto T, et al. Herpes Simplex Virus 1 infection: misleading findings in an infant with disseminated disease. New Microbiol 2013;36(3):307-313

7 Vanderpluym C, Tawfik G, Hervas-Malo M, Lacaze-Masmonteil T, Kellner J, Robinson JL. Empiric acyclovir for neonatal herpes simplex virus infection. J Matern Fetal Neonatal Med 2012; 25(8):1278-1282

8 Wada K, Mizoguchi S, Ito Y, et al. Multiplex real-time PCR for the simultaneous detection of herpes simplex virus, human herpesvirus 6, and human herpesvirus 7. Microbiol Immunol 2009;53(1):22-29

9 Fukazawa M, Hoshina T, Nanishi E, et al. Neonatal hemophagocytic lymphohistiocytosis associated with a vertical transmission of coxsackievirus B1. J Infect Chemother 2013;19(6):1210-1213

10 Yamada K, Yamamoto Y, Uchiyama A, et al. Successful treatment of neonatal herpes simplex-type 1 infection complicated by hemophagocytic lymphohistiocytosis and acute liver failure. Tohoku J Exp Med 2008;214(1):1-5

11 Nagamori T, Koyano S, Asai Y, et al. Sequential changes in pathophysiology of systemic inflammatory response in a disseminated neonatal herpes simplex virus (HSV) infection. J Clin Virol 2012; 53(3):265-267

12 Scoble JA, Underwood MA. Whole blood polymerase chain reaction in a neonate with disseminated herpes simplex virus infection and liver failure. AJP Rep 2013;3(2):67-70 